\title{
Direct myocardial ischemia imaging with exercise ${ }^{18} \mathrm{FDG}$
}

\author{
Diwakar Jain, MD, FACC, FRCP, FASNC, a and Zuo-Xiang He, MD \\ ${ }^{a}$ Cardiovascular Nuclear Imaging Laboratory, New York Medical College, Westchester Medical \\ Center, Valhalla, NY \\ ${ }^{b}$ Department of Nuclear Medicine, Cardiovascular Institute and Fu Wai Hospital, Chinese \\ Academy of Medical Sciences \& Peking Union Medical College, Beijing, China
}

doi: $10.1007 / \mathrm{s} 12350-014-0001-7$

\section{See related article, pp. 98-110}

Myocardial perfusion imaging (MPI) using SPECT or PET tracers has dominated the field of non-invasive evaluation and work-up of patients with known or suspected coronary artery disease (CAD) ${ }^{1-3}$ This technique has been around for over four decades now. This longevity of MPI is perhaps the most important testimonial of the versatility, robustness, reliability, and safety of this technique. Constant refinements, innovations, research and development in the fields of perfusion tracers, pharmacological stress agents, instrumentation and software for analysis, and interpretation have contributed to MPI remaining relevant to the rapidly progressing field of CAD. Databases including hundreds of thousand of patients spanning over several decades have been used to probe its diagnostic and prognostic utility in a variety of patient populations. ${ }^{3,4}$ MPI provides very powerful diagnostic as well as prognostic information in a very wide spectrum of patients: patients with clinically suspected CAD, patients with established $\mathrm{CAD}$, patients with recent or prior myocardial infarction, patients who have undergone revascularization procedures, patients with heart failure as well as patients with multiple co-morbidities and organ failures and those unable to exercise. ${ }^{3,4}$ The success of scintigraphic MPI has encouraged all other imaging modalities:

Reprint requests: Diwakar Jain, MD, FACC, FRCP, FASNC, Cardiovascular Nuclear Imaging Laboratory, New York Medical College, Westchester Medical Center, Macy Pavilion 111, 100 Woods Road, Valhalla, NY 10595;dj2700@gmail.com

J Nucl Cardiol 2015;22:111-4.

$1071-3581 / \$ 34.00$

Copyright (C) 2014 American Society of Nuclear Cardiology. echocardiography, magnetic resonance imaging, and CT angiography to follow its footprint to develop their MPI techniques. ${ }^{5,6}$ Although all of these imaging modalities have succeeded to some extent in developing techniques for MPI, yet these techniques remain somewhat cumbersome, complicated, technically challenging, limited only to very carefully selected patient populations and are far from being ready for routine clinical use. But most importantly, none of these techniques are currently able or likely to overcome the limitations of scintigraphic MPI.

Although, the sensitivity and specificity of scintigraphic stress-rest MPI are good, yet they are not ideal. The sensitivity of MPI for the detection of individual vessels with significant CAD is suboptimal. Artifacts due to attenuation, tracer activity in the adjoining organs and other technical factors remain important causes for suboptimal image quality and false positive studies. So what else can be done to overcome these limitations? ${ }^{7}$ Perhaps, it is time to think beyond the paradigm of MPI: After all, MPI is based upon the concept of imaging myocardial perfusion at rest or baseline and again after maximum coronary hyperemia induced by exercise or pharmacological stress. The areas of relative hypoperfusion on stress in comparison to rest represent myocardial ischemia. So why not image myocardial ischemia directly $?^{8}$ In fact, a major strength of scintigraphic imaging is its ability to image a very wide array of biological, metabolic, and biochemical processes, cell membrane receptors and transporters in intact organisms under various physiological conditions. This requires an intricate understanding of the biological process under evaluation and development and radiolabeling of suitable ligands to investigate this process. Using this principle, investigators have been able to image apoptosis, necrosis, angiogenesis, matrix metalloproteinases, and gene expression in experimental animal models as well as in human. ${ }^{9-15}$ The same principles can be applied for myocardial ischemia imaging as well. Myocardial 
ischemia is associated with very distinctive and profound changes in local and regional $\mathrm{pH}, \mathrm{pO}_{2}$, substrate utilization, and metabolism. Some of these changes are evanescent, whereas some others persist long enough to facilitate imaging. Normal myocardium can utilize a wide spectrum of substrates for energy production such as free fatty acids, glucose, lactate, ketone bodies, amino acids, etc. with the free fatty acids and glucose being the predominant components. The relative proportion of free fatty acids and glucose uptake and utilization varies with their blood levels, metabolic milieu (fed or fasting state), insulin, and catecholamine levels. ${ }^{16-18}$ Free fatty acid metabolism is obligatory aerobic. Glucose metabolism is a two-stepped process, the first step (glycolysis) where glucose is converted to pyruvate is anaerobic, whereas the second step whereby pyruvate enters Kreb's cycle and is metabolized to carbon dioxide and water is aerobic. Under normal conditions, free fatty acids are the predominant source of energy production, but with the onset of myocardial ischemia, free fatty acid uptake diminishes substantially, and glycolysis becomes the predominant source of energy. However, glycolysis being a relatively inefficient source of energy production, only a multifold increase in glucose uptake and glycolysis can sustain ischemic myocardium. This is mediated by an immediate translocation of highly specialized glucose transporters (GLUTs) from cytosol to the cell membrane with the onset of ischemia. Interestingly, once translocated to the cell membrane, GLUTs persist in the cell membrane for several hours or even longer even after the resolution of ischemia. ${ }^{19}$ Therefore, the metabolic accompaniments of myocardial ischemia can potentially be used as surrogates for imaging myocardial ischemia. The ultimate success of imaging a biological phenomenon by scintigraphic imaging is dependent upon a number of factors such as the abundance of the target signal, specificity of this signal for that particular biological phenomenon, duration for the expression of this signal, and availability of suitable and specific ligands or probes and radiolabeling of such probes with commonly available radiotracers. Whereas, any number of factors may preclude a successful and clinically usable and relevant imaging of a biological phenomenon, it is definitely worth a try. ${ }^{18} \mathrm{FDG}$, a radiolabeled glucose analogue has been evaluated is several relatively small experimental animal as well as clinical studies as a potential myocardial ischemia imaging agent with highly encouraging results. ${ }^{20-32}$ Although earlier studies indicated its feasibility, the clinical potential of this technique was appreciated only after it was possible to precisely localize any observed or perceived increased ${ }^{18} \mathrm{FDG}$ uptake to the myocardium either by simultaneous perfusion and ${ }^{18} \mathrm{FDG}$ imaging using PET imaging capable gamma cameras or by PETCT imaging. ${ }^{24,28}$

In this issue of the journal Sasikumar et al have presented their preliminary data on successful use of ${ }^{18}$ FDG PET-CT for imaging of exercise-induced myocardial ischemia. ${ }^{33}$ They performed exercise-rest SPECT MPI and exercise ${ }^{18}$ FDG PET-CT imaging and coronary angiography in 45 patients with suspected CAD. Of these, 27 patients had $\geq 50 \%$ narrowing of $\geq 1$ coronary artery and the remaining 18 had no significant CAD. The overall sensitivity and specificity of MPI were $56 \%$ and $72 \%$ compared to $96 \%$ and $44 \%$ for exercise ${ }^{18} \mathrm{FDG}(P=.0004)$. This difference narrowed when the threshold of significant $\mathrm{CAD}$ was changed to $\geq 70$ narrowing (MPI sensitivity and specificity $90 \%$ and $72 \%$ vs $100 \%$ and $44 \%$, respectively, for exercise $\left.{ }^{18} \mathrm{FDG}, P=\mathrm{ns}\right)$. Furthermore, the sensitivity of MPI for the detection of individual vascular territories with $\geq 50 \%$ narrowing was $24 \%$ for LAD, $27 \%$ for LCx, and $86 \%$ for the RCA, whereas the corresponding sensitivities for exercise ${ }^{18}$ FDG were $71 \%, 80 \%$ and $57 \%$, respectively.

Despite the limitations of relatively small sample size, this study adds significantly to the extant literature on exercise ${ }^{18} \mathrm{FDG}$ for the detection of CAD. Once again, this study confirms the previous observations of significantly higher sensitivity of exercise ${ }^{18} \mathrm{FDG}$ for the detection of CAD compared to exercise-rest MPI. However, this study also raises several important questions and issues. The overall sensitivity of exercise MPI was substantially lower compared to the published literature in this study. The authors have not provided any explanation for this. A low specificity of exercise ${ }^{18}$ FDG $(44 \%)$ is also very concerning. Notably $10 / 18$ patients with no significant CAD had increased regional myocardial ${ }^{18}$ FDG uptake. This requires a very careful thinking. A small proportion of these false positive cases also had reversible perfusion abnormalities, and/or ST segment depression on exercise. Nine of the ten patients had hypertension. This may highlight the limitations of using 50\% luminal narrowing on coronary angiography as the gold standard for comparing other diagnostic modalities. Nevertheless, this still indicates an unacceptably low specificity of exercise ${ }^{18}$ FDG imaging. Interestingly, nearly all these false positive exercise ${ }^{18} \mathrm{FDG}$ studies were due to increased ${ }^{18} \mathrm{FDG}$ uptake in the lateral wall. The authors performed a semi-quantitative visual scoring of the intensity of myocardial ${ }^{18}$ FDG uptake, similar to what was performed by Dou et al while performing ${ }^{18}$ FDG SPECT imaging. ${ }^{29}$ They did not perform quantitative imaging readily available with PET imaging. Perhaps, a simple standardized uptake value (SUV) may be able to define a threshold 
above which the uptake in the lateral wall is indeed indicative of ischemia.

Despite the limitations of a small sample size and a lack of quantitative analysis, the current study greatly enhances our understanding of the concept of direct myocardial ischemia. Direct myocardial ischemia imaging with exercise ${ }^{18} \mathrm{FDG}$ can be a very useful and highly promising diagnostic option, at least in a subgroup of patients with CAD. Perhaps, it is the right time for considering a large multicenter study to evaluate the role of exercise ${ }^{18}$ FDG imaging in the management of patients with CAD. A lot more information is needed about its specificity and any confounding effects of the presence of hypertension, diabetes, other myocardial diseases, and treatment with cardiac as well as noncardiac medications. An ability to image myocardial ischemia does indeed represent a unique and major strength of the molecular cardiovascular nuclear imaging surpassing all other imaging modalities.

\section{References}

1. Zaret BL, Strauss HW, Martin ND, Wells HP Jr, Flamm MD Jr. Noninvasive regional myocardial perfusion with radioactive potassium: Study of patients at rest, with exercise and during angina pectoris. N Engl J Med 1973;19:809-12.

2. Wackers FJ, Sokole EB, Samson G, Schoot JB, Lie KI, Liem KL, et al. Value and limitations of thallium-201 scintigraphy in the acute phase of myocardial infarction. N Engl J Med 1976;295:1-5.

3. Jain D, Zaret BL. Chapter: Nuclear cardiology. In: Rosendorf C, editor. Essential cardiology: Principles \& practices. 3rd ed. New York: Springer; 2013.

4. Cremer P, Hachamovitch R, Tamarappoo B. Clinical decision making with myocardial perfusion imaging in patients with known or suspected coronary artery disease. Semin Nucl Med 2014; 44:320-9.

5. Senior R, Monaghan M, Main ML. Detection of coronary artery disease with perfusion stress echocardiography using a novel ultrasound imaging agent: Two phase 3 international trials in comparison with radionuclide imaging. Eur J Echocardiogr 2009; 10:26-35.

6. Bamberg F, Marcus RP, Becker A, Hildebrandt K, Bauner K, Schwarz F, et al. Dynamic myocardial CT perfusion imaging for evaluation of myocardial ischemia as determined by MR imaging. JACC Cardiovasc Imaging 2014;7:267-77.

7. Zaret BL. Pursuit of the ideal perfusion agent. J Nucl Cardiol 2002;9:149-50 (editorial).

8. Jain D, McNulty PH. Exercise-induced myocardial ischemia: Can this be imaged with F-18-fluorodeoxyglucose? J Nucl Cardiol 2000;7:286-288 (editorial).

9. Wolters SL, Corsten MF, Reutelingsperger CP, Narula J, Hofstra L. Cardiovascular molecular imaging of apoptosis. Eur J Nucl Med Mol Imaging 2007;34:S86-98.

10. Boersma HH, Kietselaer BL, Stolk LM, Bennaghmouch A, Hofstra L, Narula J, et al. Past, present, and future of annexin A5: From protein discovery to clinical applications. J Nucl Med 2005;46:2035-50.

11. Narula J, Kietselaer B, Hofstra L. Role of molecular imaging in defining and denying death. J Nucl Cardiol 2004;11:349-57.
12. Morrison AR, Sinusas AJ. New molecular imaging targets to characterize myocardial biology. Cardiol Clin 2009;27:329-44.

13. Jain D, Lahiri A, Raftery EB. Immunoscintigraphy for detecting acute myocardial infarction without electrocardiographic changes. Br Med J 1990;300:151-3.

14. Li F, Jiang S, Zu Y, Lee DY, Li Z. A tyrosine kinase inhibitorbased high-affinity pet radiopharmaceutical targets vascular endothelial growth factor receptor. J Nucl Med 2014;55:1525-31.

15. Avril N, Bengel FM. Defining the success of cardiac gene therapy: How can nuclear imaging contribute? Eur J Nucl Med Mol Imaging 2003;30:757-71.

16. Neely JR, Rovetto MJ, Oram JF. Myocardial utilization of carbohydrates and lipids. Prog Cardiovasc Dis 1972;15:289-329.

17. Liedtke AJ. Alterations of carbohydrate and lipid metabolism in the acutely ischemic heart. Prog Cardiovasc Dis 1981;23:321-36.

18. Young LH, Coven DL, Russell RR 3rd. Cellular and molecular regulation of cardiac glucose transport. J Nucl Cardiol 2000;7:26776.

19. Sun D, Nguyen N, Degrado T, Schwaiger M, Brosius FC. Ischemia induces translocation of the insulin-responsive glucose transporter GLUT 4 to the plasma membrane of cardiac myocytes. Circulation 1994;89:793-8.

20. Schelbert HR, Henze E, Phelps ME, Kuhl DE. Assessment of regional myocardial ischemia by positron-emission computed tomography. Am Heart J 1982;103:588-97.

21. Camici P, Araujo LI, Spinks T, Lammertsma AA, Kaski JC, Shea $\mathrm{MJ}$, et al. Increased uptake of ${ }^{18} \mathrm{~F}$-fluorodeoxyglucose in postischemic myocardium of patients with exercise-induced angina. Circulation 1986;74:81-8.

22. Abramson BL, Ruddy TD, de Kemp RA, Laramee LA, Marquis JF, Beanlands RSB. Stress perfusion/metabolic imaging: A pilot study for a potential new approach to the diagnosis of coronary artery disease in women. J Nucl Cardiol 2000;7:205-12.

23. Araujo LI, McFalls EO, Lammertsma AA, Jones T, Maseri A. Dipyridamole-induced increased glucose uptake in patients with single-vessel coronary artery disease assessed with PET. J Nucl Cardiol 2001;8:339-46.

24. He ZX, Shi RF, Wu YJ, Tian YQ, Liu XJ, Wang SW, et al. Direct imaging of exercise-induced myocardial ischemia with fluorine18-labeled deoxyglucose and Tc-99m-sestamibi in coronary artery disease. Circulation 2003;108:1208-13.

25. He ZX, Shi RF, Wu YJ, Tian YQ, Liu XJ, Wang SW, et al. Myocardial ischemia, fluorodeoxyglucose, and severity of coronary artery stenosis: The complexities of metabolic remodeling in hibernating myocardium. Circulation 2004;109:e167-70 (authors' reply to letter to the editor).

26. Arrighi JA. F-18 fluorodeoxyglucose imaging in myocardial ischemia: Beyond myocardial viability. J Nucl Cardiol 2001;8:417-20.

27. Abbott BG, Liu YH, Arrighi JA. [18F]Fluorodeoxyglucose as a memory marker of transient myocardial ischaemia. Nucl Med Commun 2007;28:89-94.

28. Jain D, He ZX. Direct imaging of myocardial ischemia: A potential new paradigm in nuclear cardiovascular imaging. J Nucl Cardiol 2008;15:617-30.

29. Dou KF, Yang MF, Yang YJ, Qiao SB, Tian YQ, Jain D, et al. Direct myocardial ischemia imaging: ${ }^{18} \mathrm{FDG}$ uptake during exercise and at rest in patients with coronary artery disease. J Nucl Med 2008;49:1986-91.

30. Jain D, He ZX, Ghanbarinia A. Exercise ${ }^{18}$ FDG imaging for the detection of CAD: What are the clinical hurdles? Curr Cardiol Rep 2010;12:170-8. doi:10.1007/s11886-010-0087-8.

31. Jain D, He ZX, Lele V, Aronow WS. Direct Myocardial ischemia imaging: A new paradigm in cardiovascular nuclear imaging. Clin Cardiol 2014 (in press). 
32. Jain D, He ZX, Lele V. Cardiac hot spot imaging with ${ }^{18}$ FDG. Semi Nucl Med 2014;44:375-85.

33. Sasikumar A, Mittal BR, Bhattacharya A, Manojkumar R. Comparison of Tc99m-tetrofosmin myocardial perfusion scintigraphy and exercise F18-FDG imaging in detection of myocardial ischemia in patients with coronary artery disease. J Nucl Cardiol (current issue). 